\title{
Development of Clustering Algorithm Applied for the EELS Analysis of Advanced Devices
}

Mu-Tuhg Chang ${ }^{1}$, Ren-Fong Cai ${ }^{2}$, Chien-Chun $\mathrm{Chen}^{3}$ and Shen-Chuan Lo ${ }^{1}$

${ }^{1}$ Industrial Technology Research Institute, Chutung, Hsinchu, Taiwan (Republic of China), ${ }^{2}$ Industrial Technology Research Institute, Hsinchu, Hsinchu, Taiwan (Republic of China), ${ }^{3}$ Department of Engineering and System Science, National Tsing Hua University, Hsinchu, Taiwan, Hsinchu, Hsinchu, Taiwan (Republic of China)

With the coming of the third age of artificial intelligence, machine learning has been successfully implemented in many fields, which changed the paradigms to feed tons of data to find the pattern that with similar features. However, limited studies are focused on the electron microscopy and corresponding spectrum applications. In this study, we develop a novel de-noising approach based on the clustering algorithm, named kMMLS, which combines the k-means clustering and the multiple linear least squares (MLLS). The kMLLS clustering routine can extract the nearly pure endmember and be applied in the region of Electron Energy Loss Spectroscopy (EELS) investigations. With using the extracted endmember as the reference spectra of MLLS fitting, we can obtain the de-noising data set from the MLLS routine.

We examined the feasibility of kMLLS clustering from experimental data. A line-scanned spectrum image of $\mathrm{Si} / \mathrm{SiO} / \mathrm{W} / \mathrm{Ta} / \mathrm{W} / \mathrm{WO} 、 \mathrm{Si} / \mathrm{SiO} / \mathrm{AlO} / \mathrm{SiO} / \mathrm{SiN}$ multi-layers and advanced memory device ( $\mathrm{SiO} / \mathrm{SiN} / \mathrm{SiO} / \mathrm{W} / \mathrm{Si} / \mathrm{SiO} / \mathrm{Si}$ ) was acquired using a JEOL JEM-ARM200F Cs-Corrected STEM with a GATAN Quantum 965 EELS camera at 200keV. The procedure of kMLLS clustering was shown in figure 1, which was described in the following. Step 1: We carried out k-means clustering to obtain the candidates (first reference spectrums) for the MLLS routine. Step 2: we performed the MLLS procedure pixel-bypixel to obtain the coefficient of each reference spectra. Step 3: the renewed reference spectrums were calculated based on the coefficients of the step 2 MLLS result. The step 2 and step 3 were repeated until no coefficients were changed under the tolerance of each reference spectrums. Finally, the commercial and kMLLS clustering results were compared in figure 2 . We can prove the kMLLS is the powerful and suitable tool to resolve the overlap problems of EELS spectrums.

Finally, the graphic user interface (App) was developed using python interface. It is easy and friend for the users of materials characterization. We can package the total function into the one software product for most material characterizations. 


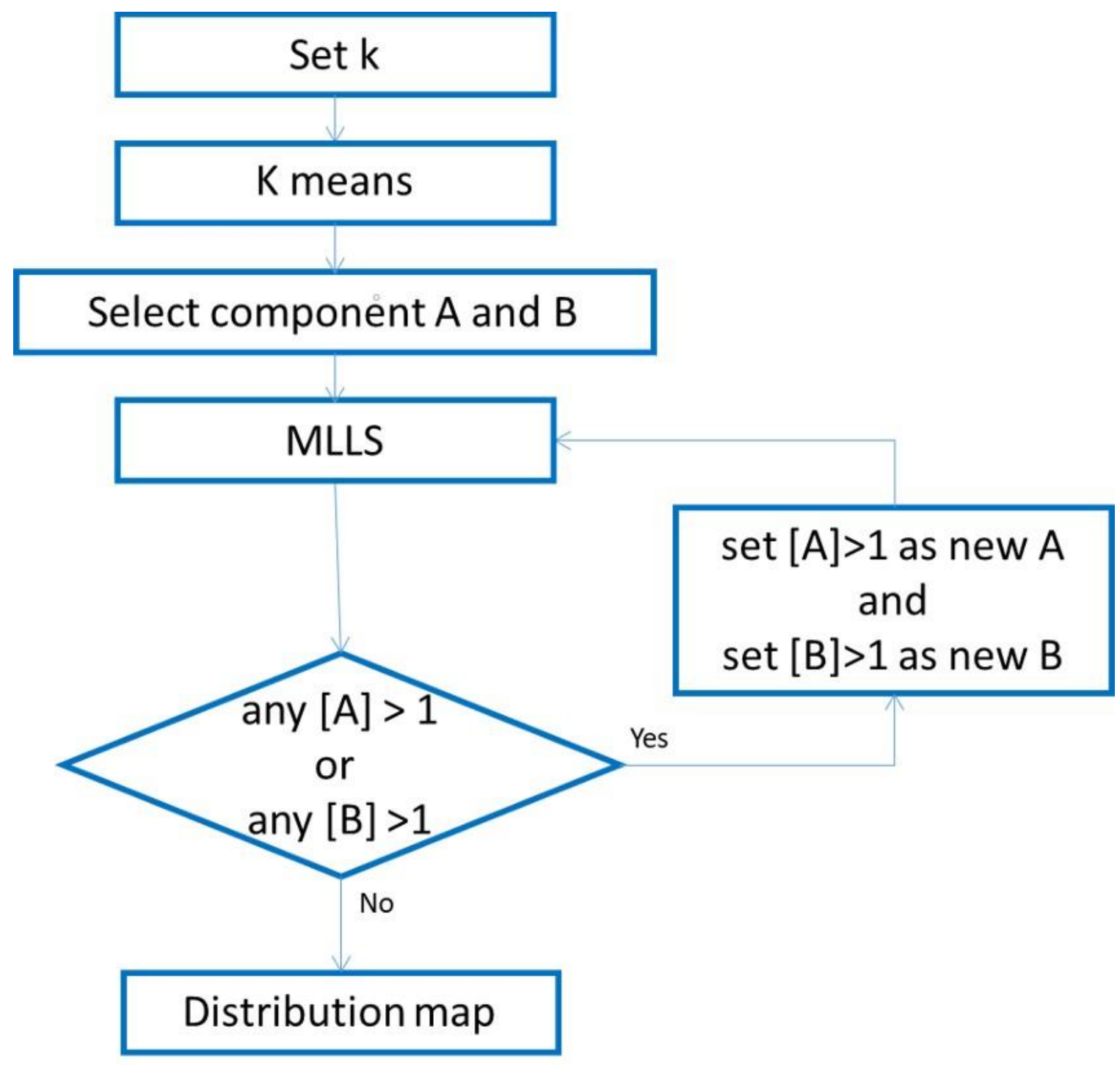

Figure 1. the procedure of kMLLS clustering 


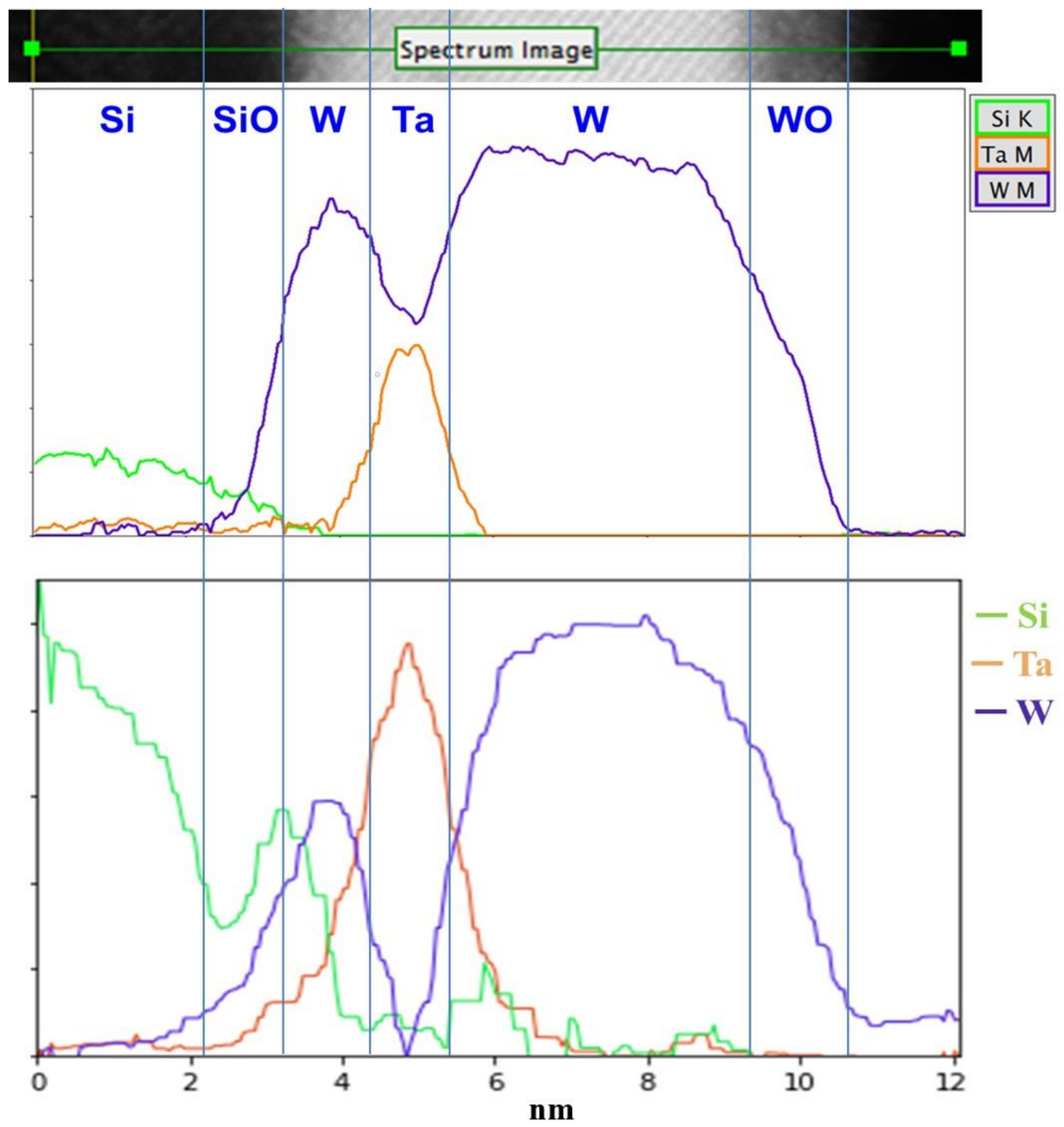

Figure 2. the comparisons of commercial and kMLLS clustering results

\section{References}

1. P.M.Voyles, CurrentOpinioninSolidStateandMaterialsScience21141(2017).

2.

S.RaschkaandV.Mirjalili,PythonMachineLearning:MachineLearningandDeepLearningwithPython,scikit -learn,andTensorFlow2ndED,Packt,Birmingham(2017).

3. A.Belianinovetal.,Nat.Commun.67801(2015). 